\title{
Adverse ocular effects associated with niacin therapy
}

\author{
F W Fraunfelder, F T Fraunfelder, D R Illingworth
}

\begin{abstract}
In a retrospective survey of patients taking medication for hyperlipidaemia, those taking niacin (nicotinic acid) were more likely $(p<0 \cdot 05)$ to report sicca syndromes, blurred vision, eyelid oedema, and macular oedema compared with those who never took niacin. Additionally, $7 \%$ of those taking niacin discontinued the drug owing to adverse ocular side effects, while none of the other lipid lowering agents were found to cause these side effects $(p=0 \cdot 016)$. Data from spontaneous reporting systems support a possible association of decreased vision, cystoid macular oedema, sicca-like symptoms, discoloration of the eyelids with or without periorbital or eyelid oedema, proptosis, loss of eyebrow or eyelashes, and superficial punctate keratitis with the use of niacin in high doses. Decreased vision may be marked, and if the drug is not discontinued, may progress to cystoid macular oedema. All ocular side effects listed above are reversible if the association with niacin is recognised and the drug is discontinued; both the incidence and severity of the ocular side effects seem to be dose dependent.
\end{abstract}

(Br f Ophthalmol 1995; 79: 54-56)

Niacin (nicotinic acid) can be purchased without prescription from health food outlets and pharmacies and is used to improve circulation, as a vitamin supplement, or as a cholesterol lowering agent. While doctors have known for some time that niacin causes significant ocular side effects, to the point where patients may have to discontinue this medication, the literature only contains seven cases ${ }^{1-3}$ of cystoid macular oedema and three cases of blurred vision. ${ }^{3-5}$ The purpose of this paper is to outline the possible and probable ocular side effects associated with the use of niacin and to determine if the frequency of these events differs from those taking other lipid lowering agents.

Sciences University,

Portland, Oregon,

USA

F W Fraunfelder

F T Fraunfelder

D R Illingworth

Correspondence to: F T Fraunfelder, MD, Casey Eye Institute, Oregon, Sciences University, Department of

Ophthalmology, $3375 \mathrm{SW}$ Portland, OR 97201, USA

Accepted for publication 5 September 1994 A survey developed by the epidemiology department at the Oregon Health Sciences University was mailed to patients who were being followed by one of the authors (DRI) in the lipid disorder clinic at the Oregon Health Sciences University. The questionnaire consisted of 20 questions focusing on ocular related side effects that could possibly be
Questionnaires were sent to two groups of 116 patients. One group consisted of patients who were being treated with niacin or had been on this agent at a dose of $3 \mathrm{~g}$ or more per day for the treatment of hyperlipidaemia. ${ }^{6}$ The second 'control group' was never on niacin, but was on other lipid lowering agents.

Although the choice of manufacturers of niacin was random, all patients were taking crystalline preparations of this drug rather than time release formulations which are more hepatotoxic. ${ }^{6}$ This retrospective survey emphasised the potential ocular side effects. Space adjacent to the questions was provided to encourage comments. If necessary, further contact was initiated with patients to clarify response.

In addition, we conducted a review of the world literature, investigated physicians' case reports sent to the National Registry of DrugInduced Ocular Side Effects at the Casey Eye Institute, Portland, Oregon, and obtained data from the FDA spontaneous drug reporting system to provide a broader scope of the side effects commonly reported by those using this drug.

Data were analysed using Statistical Analysis Software (SAS Institute, Cary, North Carolina). Odds ratios were calculated to estimate the association between treatment and adverse effect. Because of the small numbers of respondents experiencing some of the adverse effects, calculation of odds ratios was not always possible. In these cases, the Fisher's exact test was used for significance testing. Since sex of respondents differed significantly between cases and controls, multiple logistic regression was used to estimate the odds ratios adjusted for sex and sex by therapy interaction. However, in only one situation was sex significantly associated with adverse outcome (seeing halos). Adjusting for sex and sex by therapy interaction did not alter the significance or direction of the univariate associations.

\section{Results}

One hundred and two $(88 \%)$ cases and 88 $(76 \%)$ controls returned the questionnaire. Of the 102 cases, $42(41 \%)$ were females compared with 48 of $88(55 \%)$ of the controls $(p=0.019)$. The mean age of the respondents was 56 years (range 32-84) and did not differ significantly between cases and controls. attributed to the hyperlipidaemia therapy.
BLURRED VISION

Frequencies of adverse effects reported by cases and controls in the retrospective survey 
Table 1 Frequency of adverse effects by therapy

\begin{tabular}{|c|c|c|c|c|c|c|}
\hline \multirow[b]{2}{*}{ Adverse effect } & \multicolumn{2}{|c|}{ Cases } & \multicolumn{2}{|c|}{ Controls } & \multirow{2}{*}{$\begin{array}{l}\text { Odds ratio } \\
(95 \% \mathrm{CI})\end{array}$} & \multirow{2}{*}{$\begin{array}{l}\text { Fisher's exact } \\
\text { p value }\end{array}$} \\
\hline & $n$ & $\%$ & $n$ & $\%$ & & \\
\hline Dryness & 20 & 20 & 6 & 7 & $3 \cdot 4(1 \cdot 3,8 \cdot 8)$ & 0.011 \\
\hline Blurred vision & 26 & 25 & 5 & 6 & $5 \cdot 7(2 \cdot 1,15 \cdot 7)$ & 0.001 \\
\hline Double vision & 2 & 2 & 0 & 0 & $-\star$ & 0.500 \\
\hline Discoloration & 8 & 8 & 3 & 3 & $2 \cdot 4(0 \cdot 6,9 \cdot 4)$ & $0 \cdot 227$ \\
\hline Swelling & 10 & 10 & 0 & 0 & $-^{\star}$ & 0.002 \\
\hline Loss of lashes & 2 & 2 & 0 & 0 & $-\star$ & $0 \cdot 500$ \\
\hline Halos & 7 & 7 & 2 & 2 & $3 \cdot 2(0 \cdot 6,15 \cdot 8)$ & $0 \cdot 179$ \\
\hline Allergic reaction & 3 & 3 & 2 & 2 & $2 \cdot 2(0 \cdot 5,11 \cdot 7)$ & 0.454 \\
\hline Discontinued for ocular reasons & 7 & 7 & 0 & 0 & $-^{\star}$ & 0.016 \\
\hline Adverse ocular symptoms & 8 & 8 & 2 & 2 & $3 \cdot 6(0 \cdot 7,17 \cdot 3)$ & $0 \cdot 113$ \\
\hline Other side effects & 63 & 62 & 16 & 18 & $7 \cdot 2(3 \cdot 7,14 \cdot 1)$ & $<0.0001$ \\
\hline
\end{tabular}

*Unable to estimate because of zero cells.

are displayed in Table 1 . Twenty six (25\%) of the cases reported blurred vision compared with five $(6 \%)$ of the control respondents $(p=0.001)$. All of the cases were being treated with a minimum of $3 \mathrm{~g}$ up to a maximum of $8 \mathrm{~g}$ of niacin per day. In our opinion, four patients had symptoms which were possibly related to niacin on a macular basis. One patient had progressive blurring of vision to the point where she was unable to function. Once she stopped using niacin, within 48-72 hours, her vision returned to normal. A second patient had a similar experience, but when she decreased her dosage from $3 \mathrm{~g}$ per day to $1.5 \mathrm{~g}$ per day, her vision returned to near normal, and she continued to use the medication. A third complained of blurred vision for 1 hour after each dose of niacin. The fourth patient complained of blurred vision whenever she was on niacin; however, off this medication her vision was normal. The blurring was not significant enough for her to discontinue the medication.

In addition to the 26 niacin treated cases of blurred vision noted in the survey, three cases were reported in the literature ${ }^{3-5}$ and 18 cases were reported to the National Registry or the FDA spontaneous reporting system. Among the 21 cases in the registry and in the literature, 12 were male, seven were female, and in two the sex was not reported. The average dose of niacin was $2.2 \mathrm{~g}$ per day, with a duration of therapy varying from 6 weeks to 1 year. Follow up, although incomplete, reported complete resolution of visual symptoms after discontinuing niacin.

\section{DRY EYES}

In the retrospective survey, $20 \%$ (20 cases) of the patients felt that they had dry eyes or decreased tears after starting niacin. This compares with $7 \%$ in the control group (odds ratio $=3 \cdot 4 ; 95 \% \mathrm{CI}=1 \cdot 3-8 \cdot 8)$. However, 14 of the cases were already aware of ocular sicca problems, and their symptoms became worse on niacin. The remaining six felt that the niacin caused them to develop sicca.

SWELLING OF EYELIDS

In the survey group, 10 cases $(10 \%)$ complained of swelling of their eyelids, usually worse in the morning. None of the controls reported such an effect $(p=0.002)$.

\section{OTHER EFFECTS POSSIBLY ASSOCIATED WITH}

NIACIN USE

Eight per cent of cases and 3\% of controls reported a greyish appearance of their eyelids, usually worse in the morning. Seven female subjects and two controls saw white halos at night, especially while driving. Two subjects complained of intermittent diplopia on niacin, however, neither stopped the medication because of this.

Other possible niacin related side effects in the national registry include two cases of episcleritis, two cases of reversible loss of eyelashes and eyebrows, three cases of proptosis, and four cases of transitory superficial punctate keratitis.

\section{CYSTOID MACULOPATHY}

Two of the patients surveyed discontinued use of niacin owing to symptoms suggestive of cystoid macular oedema. World literature has seven cases ${ }^{1-3}$ and the national registry has five cases of cystoid macular oedema. Ten of these patients were male, one female, and the sex was not reported in one case. The average dosage of niacin was $3.6 \mathrm{~g}$ per day with a duration of therapy ranging from 1 to 36 months. In nine patients, ocular symptoms resolved within a period of 1 week to 5 months after niacin therapy had been discontinued; long term follow up was not obtained in the other three patients.

\section{REASONS FOR DISCONTINUING NIACIN}

In the retrospective survey, all seven cases who discontinued niacin therapy (six patients) or reduced the dose (one patient) because of ocular symptoms were male. Among the six patients who stropped the drug, two stopped because of symptoms suggestive of cystoid macular oedema, whereas the four remaining patients had significant symptoms of blurred vision. The decision to stop niacin was because of concurrent symptoms of lid oedema and aggravated sicca in two patients. One patient decreased his dosage (from $3 \mathrm{~g}$ to $1.5 \mathrm{~g}$ ), which controlled his blurred vision to a tolerable level.

\section{Discussion}

This is the first comprehensive report of ocular side effects associated with niacin usage. It is surprising that so little has been written on the ocular side effects of this widely used drug. This may be, in part, because all of the observed side effects appear to be reversible, and doctors do not feel the need for ophthalmic consultation.

The most serious niacin related ocular adverse effect, cystoid macular oedema, was first described by Gass. ${ }^{1}$ On the basis of the known cases of niacin related maculopathy, there is a 10:1 male:female ratio. Most cases were in their third to fifth decade of life and were being treated with an average dose of $3.6 \mathrm{~g}$ of niacin per day. The patients' visual symptoms resolved in a few months, and to our 
knowledge all visual symptoms resolved if the niacin was discontinued. The aetiology of niacin's effect on the macula is unknown, although there is a theory of prostaglandin induced toxic responses on the Muller cells or intracellular accumulation of fluid secondary to some as yet undocumented derangement of intracellular metabolism. ${ }^{9}$ We feel that a number of the 47 cases of blurred vision in the niacin therapy group may be due to macular disturbance. This seems to be, in part, a dose related event since some patients were able to decrease their dosage to control their decreased vision. In a few patients with marked bilateral decreases in vision, symptoms resolved in 1-48 hours after the drug was discontinued. Decreased vision could occur at a lower dosage than cystoid oedema, with an equal male to female ratio predilection in this series. This does not reflect a higher frequency of hyperlipidaemia in men compared with women, or a greater use of hyperlipidaemia drugs in men. ${ }^{78}$

No cases of aggravation of ocular sicca were reported in the spontaneous reporting series; in contrast, this side effect was reported by $20 \%$ of the patients in our retrospective series. Since, in our opinion, patients with familial hypercholesterolaemia have a high incidence of ocular sicca and Sjogren's syndrome, these patients possibly complained of aggravation of their sicca rather than niacin causing sicca. We feel a possible explanation is that this vitamin may be secreted and concentrated in human tears, thereby irritating an already dry eye. This may also be the reason for the four reported cases of superficial punctate keratitis. This is not unlike vitamin $\mathrm{A}$ which has been shown to be secreted in the tears. ${ }^{10}$

We have no good explanation for the white halo around lights, lid swelling or lid discoloration with a clear predominance in females. The cases of diplopia were intermittent and did not require discontinuation of drug therapy. The cases of loss of eyelashes or eyebrows may or may not be related to niacin, but alopecia secondary to drugs is not uncommon. It is of interest that all of the approximately $7 \%$ of patients who stopped using niacin were males and stopped the drug because of visual changes.

Niacin can cause ocular signs and symptoms, but they all seem to be reversible. They also seem to be dose related, so if the patient wishes to continue this therapy, it may be feasible to reduce the dose of drug. Niacin appears to have a direct effect on the macula, and if blurring of vision occurs, macular oedema should be included in the differential diagnosis and the drug promptly stopped. If sicca is aggravated, this may be relieved by increasing the frequency of artificial tears. Periorbital and lid oedema or discoloration also seem to be dose related and cause cosmetic problems. Niacin is an effective agent for the treatment of many patients with hyperlipidaemia ${ }^{10}$ and, from an ocular point of view, also appears to be a relatively safe medication. However, for optimal efficacy and safety, the side effect profile of niacin needs to be fully appreciated; in some cases the drug may need to be discontinued because of ocular side effects.

This study was supported, in part, by a grant from Research to Prevent Blindness.

1 Gass JDM. Nicotinic acid maculopathy. Am $\mathcal{f}$ Ophthalmo 1973; 76: 500-10.

2 Chazin BJ. Effect of nicotinic acid on blood cholesterol. Geriatrics 1960; 15: 423.

3 Millay RH, Klein ML, Illingworth DR. Niacin maculopathy. Ophthalmology 1988; 95: 930.

4 Harris JL. Toxic amblyopia associated with administration nicotinic acid. Am f Ophthalmol 1963; 55: 133.

5 Parsons WB Jr, Flinn JH. Reduction in elevated blood cholesterol levels by large doses of nicotinic acid. $\mathcal{J} A M A$ 1957; 165: 234-8.

6 Larsen ML, Illingworth DR. Triglyceride lowering agents, fibrates and nicotinic acid. Current Opinion in Lipidology 1993; 4: 34-40.

7 The Expert Panel. Report of the national cholesterol education program expert panel on detection, evaluation and treatment of high blood cholesterol in adults. Arch Intern Med 1988; 148: 36-69.

8 Wysowski DK, Kennedy DL, Gross TP. Prescribed use of cholesterol-lowering drugs in the United States 1978-1988. $₹ A M A$ 1990; 263: 2185-8.

9 Jampol LM. Niacin maculopathy. Author's reply RH Millay. Ophthalmology 1988; 95: 1704-5.

10 Ubels JL, MacRae SM. Vitamin A is present in retino in tears of humans and rabbits. Curr Eye Res 1984; 3 815. 